\title{
THE NEW BETTER THAN USED FAILURE RATE CLASS OF LIFE DISTRIBUTION
}

\author{
A. M. ABOUAMMOH, ${ }^{*}$ AND \\ A. N. AHMED,${ }^{*}$ King Saud University
}

\begin{abstract}
A new concept of ageing distribution, namely new better than used in failure rate (NBUFR), is introduced. Different properties of the NBUFR class and its dual class are presented. Its relations to other classes of life distributions are investigated. Finally, NBUFR survival under shock models is discussed.
\end{abstract}

\section{Introduction}

Let $T$ be a non-negative random variable representing equipment failure time with failure distribution $F(t)=P(T \leqq t)$, survival function $\bar{F}(t)=1-F(t)$ and density function $f$ (if it exists). The corresponding failure rate $r(t)$, when the distribution is absolutely continuous (a.e. [Lebesgue]), is defined by

$$
r(t)=\lim _{\delta t \rightarrow 0} \frac{1}{\delta t} P(T \leqq t+\delta t \mid T>t) .
$$

The random variable $T$ is said to have increasing failure rate (IFR) or increasing failure rate average (IFRA) if $r(t)$ or $t^{-1} \int_{0}^{t} r(x) d x$ is increasing, respectively. Other well-known classes are decreasing mean residual life (DMRL), new better than used (NBU), new better than used in expectation (NBUE) and new better than used average in failure rate (NBAFR). For definitions of these classes and their duals see Bryson and Siddiqui (1969), Barlow and Proschan (1981) and Loh (1984).

Now we introduce the following.

(1.1) Definition. An absolutely continuous probability distribution $F$ on $[0, \infty)$ for which $F(x) / x$ has a limit as $x \rightarrow 0$ from above is said to be NBUFR (NWUFR) if there exists a version $r$ of the failure function such that

$$
\bar{r}(0) \leqq(\geqq) r(t),
$$

where

$$
\bar{r}(0)=\lim _{t \rightarrow 0+} t^{-1} \int_{0}^{t} r(x) d x .
$$

The next definition is mainly due to Loh (1984).

(1.2) Definition. An absolutely continuous probability distribution function $F$ on $(0, \infty)$ for which $F(x) / x$ has a limit as $x \rightarrow 0$ from above is said to be NBAFR (NWAFR) if

$$
\bar{r}(0) \leqq(\geqq) t^{-1} \int_{0}^{t} r(x) d x \text { for } t>0,
$$

where $\bar{r}(0)$ is as in Definition (1.1).

Received 6 January 1987; revision received 28 September 1987.

* Postal address: Department of Statistics, College of Science, King Saud University, P.O. Box 2455, Riyadh 11451, Saudi Arabia. 
It is noted that every NBUFR (NWUFR) distribution is NBAFR (NWAFR). Also if $F$ is an absolutely continuous distribution then for every $x, t>0, \bar{F}(x+t) \leqq \bar{F}(x) \bar{F}(t)$. This implies that

$$
\lim _{x \rightarrow 0} \frac{F(x)}{x} \leqq \lim _{x \rightarrow 0} \frac{F(x+t)-F(t)}{x(1-F(t))}
$$

This means that every NBU (NWU) distribution is NBUFR (NWUFR).

The NBUFR (NWUFR) distributions enjoy many desirable properties under several reliability operations. Also, the NBUFR class is very wide since it includes any life distribution with failure rate $\bar{r}(0)=0$. It is shown that any $k$-out-of- $n, 1 \leqq k<n$ system has the NBUFR property.

Next we note the following.

(1.3) Remark. An absolutely continuous distribution $F$ on $[0, \infty)$ for which $F(x) / x$ has a limit as $x \rightarrow 0$ from above has the NBUFR property iff $\bar{F}(x+t) \leqq \exp (-\bar{r}(0) t) F(x)$ for all $x, t \geqq 0$.

(1.4) Remark. If the distribution $F$ is NBUFR and $\bar{r}_{F}(0)=\mu_{F}^{-1}(0)$, then $F$ is NBUE.

\section{Closure property}

This section contains preservation properties of NBUFR and NWUFR classes under various reliability operations.

(2.1) Theorem. If $F_{i}, i=1,2$ are probability distribution functions on $[0, \infty)$ such that $F$ is absolutely continuous with $x^{-1} F(x)$ having a finite limit superior as $x \rightarrow 0$ from above and $F_{2}(0)=0$, then $F_{1} * F_{2}$ is NBUFR.

Proof. We have that $F_{1} * F_{2}$ is an absolutely continuous distribution function on $(0, \infty)$ with $F_{1} * F_{2}(x) \leqq F_{1}(X) F_{2}(x)$ for every $x \in(0, \infty)$ yielding that $x^{-1}\left(F_{1} * F_{2}(x)\right) \rightarrow 0$ as $x \rightarrow 0$ from above (in view of the assumptions). From this it follows trivially that $F_{1} * F_{2}$ is NBUFR.

Corollary. If $F_{i}, i=1,2$ are probability distribution functions on $[0, \infty)$ that are NBUFR or simply NBAFR, then $F_{1} * F_{2}$ is NBUFR.

The NBUFR class is not preserved under convolution.

(2.2) Example. The exponential distribution $F(x)=1-\exp (-x)$ is NWUFR. The convolution $K$ of $F$ with itself is the gamma distribution of order 2, i.e. $K(x)=1-(1+x) \exp (-x)$, which has strictly increasing hazard rate. Thus $K$ is not NWUFR.

The NBUFR is not preserved under mixtures. The following example shows that a mixture of NBUFR distributions need not be NBUFR.

(2.3) Example. Let $\bar{F}_{\theta}(x)=\exp (-\theta x)$ and $\bar{G}(x)=\int_{0}^{\infty} \bar{F}_{\theta}(x) \exp (-\theta) d \theta=(1+x)^{-1}$. The density function is $g(x)=(1+x)^{-2}$ and the failure rate function $r_{G}(x)=(1+x)^{-1}$, which is strictly decreasing in $x>0$. Thus $G$ is not NBUFR.

(2.4) Theorem. If $F$ is an arbitrary mixture of $F_{\theta}, \theta \in \Theta$ where each $F_{\theta}$ is NWUFR, then $F$ is NWUFR. 
Proof. Let $r(t)$ and $r_{\theta}(t)$ be the hazard functions corresponding to $F$ and $F_{\theta}$, respectively. Then defining for convenience $-\log 0=\infty$, we see that for $t>0$

$$
\begin{aligned}
\int_{0}^{t} r(u) d u & =-\log \bar{F}(t) \\
& =-\log \left(\int_{\Theta} \bar{F}_{\theta}(t) d G(\theta)\right) \\
& \leqq \int_{\Theta}\left(-\log \bar{F}_{\theta}(t)\right) d G(\theta), \quad \text { (essentially by the Jensen inequality) } \\
& =\int_{\Theta}\left(\int_{0}^{r} r_{\theta}(u) d u\right) d G(\theta) \\
& \leqq t \int_{\Theta} \bar{r}_{\theta}(0) d G(\theta) \quad\left(\text { since each } F_{\theta}\right. \text { is NWAFR) } \\
& =t \int_{\Theta} \lim _{x \rightarrow 0+} \frac{F_{\theta}(x)}{x} d G(\theta) \\
& =t \lim _{x \rightarrow 0} \int_{\Theta} \frac{F_{\theta}(x)}{x} d G(\theta)
\end{aligned}
$$

by either Fatou's lemma or Lebesgue dominated convergence theorem since each $F_{\theta}$ is NWAFR for each $\theta$. Hence

$$
\int_{0}^{t} r(u) d u \leqq t \bar{r}(0)
$$

which implies that $F$ is NWUFR.

The following example illustrates that the NWUFR property is not preserved under mixing.

(2.5) Example. Let $F_{1}(x)=1-e^{-x}, x \geqq 0$ and

$$
F_{2}(x)=1-\exp \left(-\int_{0}^{x} \xi(y) d y, x \geqq 0\right),
$$

where $\xi$ is a continuous non-negative periodic function with unit period such that $\xi(0)=2$, $\xi(x) \leqq 2$ for all $x$ and $\int_{0}^{1} \xi(y) d y<1$. Let $F=\frac{1}{2} F_{1}+\frac{1}{2} F_{2}$. It follows that both $F_{1}$ and $F_{2}$ here are NWUFR but $F$ is not NWUFR.

\section{Poisson shock model}

Consider a device subject to shocks occurring randomly in time according to a homogeneous Poisson point process $|N(t), t \geqq 0|$ with intensity $\lambda$. Suppose that the $i$ th shock causes a random amount of damage $X_{i}, i=1,2, \ldots$ In this section we show that the device survival probability is NBUFR (NWUFR) when the probability of surviving $k(k=1,2, \ldots)$ shocks is discrete NBUFR (NWUFR).

Definition. A discrete distribution $P_{k}, 0,1, \ldots$ or its survival probability $\bar{P}_{k}=\sum_{j=k}^{\infty} p_{j}$, $k=0,1,2, \ldots$, with finite mean $\mu=\sum_{k=0}^{\infty} \bar{P}_{k+1}$, is called discrete NBUFR (NWUFR) if

$$
\bar{P}_{k+1} \leqq(\geqq) \bar{P}_{1} \bar{P}_{k} \text { for } k=0,1,2, \ldots
$$

Let $\bar{P}_{k}$ be the probability that the device survives the first $k$ shocks, where $1=\bar{P}_{0} \geqq \bar{P}_{1} \geqq$ $\bar{P}_{2} \geqq \ldots$. The survival is

$$
\bar{H}(t)=\sum_{k=0}^{\infty} \exp (-\lambda t) \frac{(\lambda t)^{k}}{k !} \bar{P}_{k} .
$$


This shock model was studied by Esary et al. (1973) for the IFR, IFRA, NBU, NBUE, and DMRL reliability classes and by Klefsjö (1981) for the HNBUE class.

(3.2) Theorem. The survival function $\bar{H}(t)$ in (3.2) is NBUFR (NWUFR) if $\left\{p_{k}\right\}_{k=0}^{\infty}$ has the discrete NBUFR (NWUFR) property in (3.1).

Proof. Under the condition (3.1) we have

$$
\begin{aligned}
r_{H}(t) & =\left(\lambda \sum_{k=0}^{\infty} P_{k} \frac{(\lambda t)^{k}}{k !} \exp (-\lambda t)\right) /\left(\sum_{k=0}^{\infty} \bar{P}_{k} \frac{(\lambda t)^{k}}{k !} \exp (-\lambda t)\right) \\
& \geqq \lambda\left(1-\bar{P}_{1}\right) \quad\left(\text { since } p_{k}=\bar{P}_{k}-\bar{P}_{k+1} \geqq\left(1-\bar{P}_{1}\right) \bar{P}_{k}\right) \\
& =\lambda p_{0}=\bar{r}_{H}(0) .
\end{aligned}
$$

The proof for the NWUFR case can be obtained by reversing the inequality in the above proof.

\section{Acknowledgement}

The authors are grateful to the referee for many constructive comments and corrections. This has led to substantial reduction in length and improvements in the presentation of the paper.

\section{References}

Barlow, R. E. and Proschan, F. (1981) Statistical Theory of Reliability and Life Testing. To Begin With, Silver Spring, MD.

Bryson, M. C. ANd Siddiqui, M. M. (1969) Some criteria for aging. J. Amer. Statist. Assoc. 23, 1472-1483.

Esary, J. D., Marshall, A. W. AND Proschan, F. (1973) Shock models and wear processes. Ann. Prob. 1, 627-649.

KLEFSJÖ, B. (1981) HNBUE survivals under some shock models. Scand. J. Statist. 8, 39-47.

LOH, W.-Y. (1984) A new generalization of the class of NBU distributions. IEEE Trans. Reliability 33, 419-422. 